\title{
An overview of the epidemiology of notifiable infectious diseases in Australia, 1991-2011
}

\author{
K. B. GIBNEY*, A. C. CHENG, R. HALL AND K. LEDER \\ Department of Epidemiology and Preventive Medicine, Monash University, The Alfred Centre, Melbourne, \\ Victoria, Australia
}

Received 24 February 2016; Final revision 28 April 2016; Accepted 5 May 2016; first published online 22 June 2016

\section{SUMMARY}

We reviewed the first 21 years (1991-2011) of Australia's National Notifiable Diseases Surveillance System (NNDSS). All nationally notified diseases (except HIV/AIDS and Creutzfeldt-Jakob disease) were analysed by disease group $(n=8)$, jurisdiction (six states and two territories), Indigenous status, age group and notification year. In total, 2421134 cases were analysed. The 10 diseases with highest notification incidence (chlamydial infection, campylobacteriosis, varicella zoster, hepatitis C, influenza, pertussis, salmonellosis, hepatitis B, gonococcal infection, and Ross River virus infection) comprised $88 \%$ of all notifications. Annual notification incidence was 591 cases/100 000, highest in the Northern Territory (2598/100 000) and in children aged $<5$ years $(698 / 100000)$. A total of $8 \cdot 4 \%$ of cases were Indigenous Australians. Notification incidence increased by $6.4 \%$ per year $(12 \%$ for sexually transmissible infections and $15 \%$ for vaccine-preventable diseases). The number of notifiable diseases also increased from 37 to 65 . The number and incidence of notifications increased throughout the study period, partly due to addition of diseases to the NNDSS and increasing availability of sensitive diagnostic tests. The most commonly notified diseases require a range of public health responses addressing highrisk sexual and drug-use behaviours, food safety and immunization. Our results highlight populations with higher notification incidence that might require tailored public health interventions.

Key words: Analysis of data, Australia, epidemiology, public health, surveillance system.

\section{INTRODUCTION}

Surveillance is the cornerstone of public health efforts to minimize morbidity and mortality resulting from preventable infectious diseases. Infectious disease surveillance was instrumental in smallpox eradication and in current efforts towards global polio eradication

\footnotetext{
* Author for correspondence: Dr K. B. Gibney, The Peter Doherty Institute for Infection and Immunity, 792 Elizabeth Street, Melbourne 3000 Victoria, Australia.

(Email: Katherine.Gibney@unimelb.edu.au)
}

and measles elimination. National surveillance systems allow examination of the epidemiological profile of important infections at a country level and provide oversight to ensure consistent reporting across jurisdictions [1].

In Australia, notification of selected infectious diseases is required by public health legislation in the six states and two territories. Each jurisdiction defines its own notification list and receives data from doctors and/or laboratories. Primary responsibility for public health action lies with the state/territory health departments. Jurisdictions forward de-identified 
notification data for cases meeting national case definitions for diseases on the National Notifiable Diseases List (NNDL) to the National Notifiable Disease Surveillance System (NNDSS), a passive surveillance system operational since 1991 .

A summary of national notifiable disease surveillance data from 1917 to 1991 highlighted the lack of consistency, detail, and methodical reporting of nationally notifiable diseases before the introduction of the NNDSS [2]. Annual reports of NNDSS data have been produced since 1991; however, trend analysis of all nationally notifiable diseases has not previously been performed. We present an overview of the epidemiology of all notifiable infectious diseases in Australia [excluding HIV/AIDS and CreutzfeldtJakob disease (CJD)] during the first 21 years of the NNDSS, with a view to highlighting diseases and population groups with greatest need of public health intervention to reduce disease incidence.

\section{METHODS}

All case notifications of nationally notifiable diseases to the NNDSS from 1 January 1991 to 31 December 2011 were analysed according to their diagnosis date [3]. HIV/AIDS and CJD are under different national surveillance systems and were excluded from this analysis [4]. Notifications were reported by disease and categorized into eight disease groups (as per the NNDL) based on mode of acquisition and/or public health strategies for control and prevention: bloodborne viral hepatitis (BBVH), gastrointestinal, other bacterial, quarantinable, sexually transmissible infections (STIs), vector-borne diseases (VBDs), vaccine-preventable diseases (VPDs), and zoonotic diseases. Diseases included in each group and the year they became notifiable are summarized in Table 1. NNDSS diseases were analysed by pathogen for hepatitis $\mathrm{B}$, hepatitis $\mathrm{C}$, rubella, syphilis, and varicella zoster.

We report the number and annual incidence of notified cases nationally and by jurisdiction. For allcause and disease-group incidence calculations, all notified cases were included and Australian Bureau of Statistics (ABS) population estimates at 30 June for each study year were used [5]. Data from the Northern Territory (NT) were excluded from both the numerator (number of cases notified) and denominator (population) of incidence calculations for 1994 due to large discrepancies between the study dataset (extracted in 2012) and online (live) NNDSS data that has undergone subsequent data cleaning [6]. For disease-specific incidence calculations, diseases notifiable both nationally and in that jurisdiction were included (Table 1) with the exceptions of hepatitis B and C (Victoria 1991-1997), hepatitis B [South Australian (SA) 1991-1995], tuberculosis (Victoria 1991), and varicella zoster (Victoria 2006-2007) due to discrepancies with online NNDSS data; the denominator comprised the combined populations for included years and jurisdictions. Relative risks (RRs) were calculated for univariate comparison of notification incidence between study sub-periods (1991-1997, 1998-2004, 2005-2011), age groups (<5, 5-19, 20-64, 65-98 years) and jurisdictions for diseases with $>400$ notifications during the 21-year study period. Three sub-periods were selected to allow more meaningful comparison between disease groups/diseases within a sub-period as well as analysis of change in notification incidence across these sub-periods for a single disease or disease group.

Average changes in annual notification incidence over the study period were investigated by Poisson regression for all diseases combined and by disease group from 1991 to 2011; for individual diseases this calculation was confined to years the disease was nationally notifiable. Tests for statistical significance were not performed as population-based data were used. To allow international comparison, age-standardized incidence rates were calculated using the WHO world standard population distribution [7].

Incidence rates for Aboriginal and Torres Strait Islander ('Indigenous') Australians were calculated for the three jurisdictions reporting Indigenous status for $>75 \%$ of notified cases [NT, SA, and Western Australia (WA)] using ABS population estimates $[8,9]$; cases with unknown Indigenous status were presumed non-Indigenous.

NNDSS data were provided by the Australian Government's Office of Health Protection on behalf of Communicable Diseases Network Australia (CDNA) jurisdictional members in March 2012 as an extract from the national data file.

\section{Ethical considerations}

The project was approved by the Monash Human Research Ethics Committee (project no. CF11/ 2357-201) and CDNA jurisdictional members. Data were analysed using Stata v. 12 (StataCorp., USA). This work did not involve human or animal experimentation. 
Table 1. Diseases included in the National Notifiable Diseases Surveillance System (NNDSS) by disease group and year introduced, Australia 1991-2011

\begin{tabular}{|c|c|c|}
\hline & Year* & Variation by jurisdiction \\
\hline \multicolumn{3}{|l|}{ Bloodborne viral hepatitis } \\
\hline Hepatitis B (newly acquired) & 1993 & 1994 in Qld and WA, 1995 in ACT \\
\hline Hepatitis B (unspecified) & 1991 & 2005 in NT \\
\hline Hepatitis C (newly acquired) & 1993 & 1995 in ACT, Tas and WA, 2005 in NT, not notifiable in Qld \\
\hline Hepatitis C (unspecified) & 1995 & Included incident cases until hepatitis $C$ newly acquired introduced \\
\hline Hepatitis D & 1999 & 2002 in WA \\
\hline Hepatitis (NEC) & 1991 & 2001 in WA. Included reports of hepatitis D and E 1991-1998 \\
\hline \multicolumn{3}{|l|}{ Gastrointestinal diseases } \\
\hline Botulism & 1992 & 1993 in SA, 1998 in NT and NSW, 2001 in WA \\
\hline Campylobacteriosis & $1991 \dagger$ & Not notifiable in NSW \\
\hline Cryptosporidiosis & 2001 & \\
\hline Haemolytic uraemic syndrome & 1999 & \\
\hline Hepatitis A & $1991 \dagger$ & \\
\hline Hepatitis E & 1999 & 2001 in WA \\
\hline Listeriosis & 1991 & 1992 in SA, 1994 in NT \\
\hline Salmonellosis (non typhoidal) & $1991 \dagger$ & \\
\hline Shiga-/Vero-toxin-producing E. coli & 1999 & 2001 in Qld and WA \\
\hline Shigellosis & 1991 & 2001 in NSW \\
\hline Typhoid fever & $1991 \dagger$ & Includes paratyphoid in NSW, Qld and Vic \\
\hline \multicolumn{3}{|l|}{ Quarantinable diseases } \\
\hline Cholera & $1991 \dagger$ & \\
\hline Highly pathogenic avian influenza (human) & 2004 & Reported under influenza in WA \\
\hline Plague & $1991 \dagger$ & \\
\hline Rabies & 1991 & 1993 in ACT, 1997 in NSW \\
\hline Severe acute respiratory syndrome & 2003 & \\
\hline Smallpox & 2004 & \\
\hline Viral haemorrhagic fever & 1991 & 1993 in ACT \\
\hline Yellow fever & $1991 \dagger$ & \\
\hline \multicolumn{3}{|l|}{ Sexually transmissible infections } \\
\hline Chancroid & 1991 & No longer nationally notifiable from 2000 \\
\hline Chlamydial infection & 1994 & 1999 in NSW \\
\hline Donovanosis & 1991 & 1993 in Tas, 2002 in NSW and SA \\
\hline Gonococcal infection & $1991 \dagger$ & \\
\hline Syphilis & 1991 & Includes syphilis $<2$ and $>2$ years/unknown duration to 2004 \\
\hline Syphilis (<2 years duration) & 2004 & \\
\hline Syphilis ( $>2$ years or unknown duration) & 2004 & Not reported in SA \\
\hline Syphilis (congenital) & $1991 \dagger$ & \\
\hline \multicolumn{3}{|l|}{ Vaccine-preventable diseases } \\
\hline Diphtheria & $1991 \dagger$ & \\
\hline Haemophilus influenzae type b & 1991 & 1994 in WA \\
\hline Influenza (laboratory confirmed) & 2001 & 2008 in SA \\
\hline Measles & $1991 \dagger$ & \\
\hline Mumps & 1995 & Not reported by Qld in 1995-96, 1999-2000 \\
\hline Pertussis & $1991 \dagger$ & \\
\hline Pneumococcal disease (invasive) & 2001 & \\
\hline Poliomyelitis & $1991 \dagger$ & \\
\hline Rubella & 1993 & 1995 in Tas \\
\hline Rubella (congenital) & $1991 \dagger$ & \\
\hline Tetanus & 1991 & 1994 in Qld \\
\hline Varicella zoster (chickenpox) & 2006 & Not notifiable in NSW \\
\hline Varicella zoster (shingles) & 2006 & Not notifiable in NSW \\
\hline Varicella zoster (unspecified) & 2006 & Not notifiable in NSW \\
\hline
\end{tabular}


Table 1 (cont.)

\begin{tabular}{|c|c|c|}
\hline & Year* & Variation by jurisdiction \\
\hline \multicolumn{3}{|l|}{ Vector-borne diseases } \\
\hline $\begin{array}{l}\text { Arbovirus infection (Not elsewhere } \\
\text { classified) }\end{array}$ & 1991 & $\begin{array}{l}\text { 1991-2000 included Japanese encephalitis, Kunjin, and Murray } \\
\text { Valley encephalitis (MVE) notifications }\end{array}$ \\
\hline Barmah Forest virus infection & 1995 & \\
\hline Dengue virus infection & 1991 & 1993 in ACT, 1995 in WA \\
\hline Japanese encephalitis virus infection & 2001 & \\
\hline Kunjn virus infection & 2001 & Reported as MVE in ACT \\
\hline Malaria & $1991 \dagger$ & \\
\hline Murray Valley encephalitis virus infection & 2001 & \\
\hline Ross River virus infection & 1993 & \\
\hline \multicolumn{3}{|l|}{ Zoonoses } \\
\hline Anthrax & 2001 & 2002 in SA \\
\hline Australian bat lyssavirus infection & 2001 & \\
\hline Brucellosis & $1991 \dagger$ & \\
\hline Hydatid infection & 1991 & No longer nationally notifiable from 2001 \\
\hline Leptospirosis & $1991 \dagger$ & \\
\hline Lyssavirus (not elsewhere classified) & 2001 & \\
\hline Ornithosis & 1991 & 2001 in NSW, Qld did not report 1991, 1997-2001 \\
\hline Q fever & $1991 \dagger$ & \\
\hline Tularaemia & 2003 & \\
\hline \multicolumn{3}{|l|}{ Other bacterial diseases } \\
\hline Legionellosis & $1991 \dagger$ & \\
\hline Leprosy & $1991 \dagger$ & \\
\hline Meningococcal disease (invasive) & $1991 \dagger$ & Includes conjunctival cases from ACT and NSW \\
\hline Tuberculosis & 1991 & \\
\hline
\end{tabular}

Source: NNDSS online (live) data and 2012 NNDSS annual report $[3,6]$.

Excludes HIV/AIDS and Creutzfeldt-Jakob disease which are notified to other surveillance systems.

ACT, Australian Capital Territory; NSW, New South Wales; NT, Northern Territory; Qld, Queensland; SA, South Australia; Tas, Tasmania; Vic, Victoria; WA, Western Australia.

* Year became nationally notifiable - listed as 1991 for diseases that were nationally notifiable when NNDSS began in 1991; diseases introduced after 1991 might have cases notified to NNDSS prior to becoming nationally notifiable.

$\dagger$ Diseases which were consistently notifiable across states for the entire study period.

\section{RESULTS}

The NNDSS contains 2421134 notified cases of 60 diseases from 1991 to 2011. STIs were most common [790 $990(32 \cdot 7 \%)$ notifications] and quarantinable diseases least common (79 notifications, all cholera) (Table 2, Fig. 1). Chlamydial infection, notifiable from 1994, was the most commonly notified disease [621 $431(26 \%)$ notifications]. The 10 pathogens with highest notification incidence were Chlamydia trachomatis, Campylobacter, varicella zoster virus, hepatitis C virus, influenza virus, Bordetella pertussis, Salmonella, hepatitis B virus, Neisseria gonorrhoeae, and Ross River virus (RRV) (Tables 3 and 4); these comprised $88 \%$ of all notifications despite campylobacteriosis and varicella zoster infection not being notifiable in New South Wales (NSW), the most populous state. Fewer than 20 notifications were received for eight diseases and no notifications were received for seven diseases (Table 3 ).

Notification numbers increased over the study period, from 43443 in 1991 (37 notifiable diseases) to 238164 in 2011 (65 notifiable diseases) (Fig. 1). The national annual notification incidence increased by an average of $6.4 \%$ per year (Fig. 2), rising from $386 / 100000$ in the earliest sub-period (1991-1997) to $853 / 100000$ in the latest sub-period (2005-2011) (Table 3). Annual notification incidence fell most markedly for rubella (average 30\% decrease/year), followed by Haemophilus influenzae type B (Hib, 25\%), measles (23\%), and donovanosis (17\%) (Table 3). Rubella and hepatitis A were among the 10 highest incidence diseases in the earliest study sub-period (Table 4). Conversely, influenza (average 33\% 
Table 2. Number, incidence, and demographics of notified cases by disease group and jurisdiction, Australia, 1991-2011

\begin{tabular}{|c|c|c|c|c|c|c|c|c|c|c|c|}
\hline & \multicolumn{2}{|c|}{ Notifications } & \multirow{2}{*}{$\begin{array}{l}\text { Age, } \\
\text { median } \\
\text { years }\end{array}$} & \multirow{2}{*}{$\begin{array}{l}\text { Male, } \\
\%\end{array}$} & \multirow{2}{*}{$\begin{array}{l}\text { Indigenous*, } \\
\%\end{array}$} & \multicolumn{2}{|c|}{$\begin{array}{l}\text { Crude incidence } \\
\text { (/100 } 000 \text { per year) }\end{array}$} & \multicolumn{2}{|c|}{$\begin{array}{l}\text { Age-standardized } \\
\text { incidence }\end{array}$} & \multicolumn{2}{|c|}{$\begin{array}{l}\text { Crude incidence, } \\
\text { Indigenous cases* }{ }^{*}\end{array}$} \\
\hline & $N$ & $(\%)$ & & & & Mean & (range) & Mean & $(95 \% \mathrm{CI})$ & Mean & (range) \\
\hline All notifications & 2421134 & $(100)$ & 27 & $51 \cdot 0$ & $8 \cdot 4$ & 591 & $(251-1092)$ & 621 & $(620-622)$ & 3764 & $(2211-4910)$ \\
\hline \multicolumn{12}{|l|}{ Disease group } \\
\hline Bloodborne viral hepatitis & 431608 & $(17 \cdot 8)$ & 34 & $61 \cdot 1$ & $3 \cdot 4$ & 106 & $(43-150)$ & 104 & $(103-104)$ & 195 & $(70-278)$ \\
\hline Gastrointestinal diseases & 518808 & $(21 \cdot 4)$ & 24 & $52 \cdot 8$ & $4 \cdot 5$ & 127 & $(88-147)$ & 136 & $(135-136)$ & 490 & $(309-819)$ \\
\hline Other bacterial diseases & 36960 & $(1 \cdot 5)$ & 37 & $55 \cdot 5$ & $5 \cdot 1$ & $9 \cdot 0$ & $(6 \cdot 4-11 \cdot 3)$ & $8 \cdot 7$ & $(8 \cdot 6-8 \cdot 8)$ & 27 & $(9-59)$ \\
\hline Quarantinable diseases & 79 & $(0 \cdot 0)$ & 42 & $50 \cdot 7$ & $2 \cdot 5$ & $0 \cdot 02$ & $(0-0.03)$ & $0 \cdot 0$ & $(0 \cdot 0-0 \cdot 0)$ & - & - \\
\hline $\begin{array}{l}\text { Sexually transmissible } \\
\text { infections }\end{array}$ & 790990 & $(32 \cdot 7)$ & 23 & $45 \cdot 7$ & $17 \cdot 8$ & 193 & $(50-428)$ & 210 & $(210-211)$ & 2758 & $(1312-3525)$ \\
\hline Vaccine-preventable diseases & 487176 & $(20 \cdot 1)$ & 25 & $47 \cdot 5$ & $3 \cdot 8$ & 119 & $(17-470)$ & 126 & $(126-126)$ & 267 & $(22-1395)$ \\
\hline Vector-borne diseases & 137817 & $(5 \cdot 7)$ & 40 & $51 \cdot 3$ & $2 \cdot 0$ & 34 & $(16-53)$ & $31 \cdot 9$ & $(31 \cdot 7-32 \cdot 0)$ & 26 & $(7-58)$ \\
\hline Zoonoses & 17696 & $(0 \cdot 7)$ & 40 & $79 \cdot 3$ & $2 \cdot 4$ & $4 \cdot 4$ & $(2 \cdot 4-6 \cdot 6)$ & $4 \cdot 1$ & $(4 \cdot 1-4 \cdot 2)$ & - & - \\
\hline \multicolumn{12}{|l|}{ Jurisdiction } \\
\hline Australian Capital Territory & 38083 & $(1 \cdot 6)$ & 26 & $51 \cdot 5$ & $0 \cdot 5$ & 560 & $(129-1098)$ & 555 & $(549-561)$ & & \\
\hline New South Wales & 584382 & $(24 \cdot 1)$ & 29 & $53 \cdot 7$ & $1 \cdot 8$ & 430 & $(114-812)$ & 449 & $(448-450)$ & & \\
\hline Northern Territory & 110930 & $(4 \cdot 6)$ & 23 & $47 \cdot 9$ & $58 \cdot 1$ & 2598 & $(1824-3653)$ & 2512 & $(2497-2523)$ & 5121 & (3609-7204) \\
\hline Queensland & 644556 & $(26 \cdot 6)$ & 26 & $49 \cdot 1$ & $9 \cdot 7$ & 850 & $(491-1504)$ & 880 & $(877-882)$ & & \\
\hline South Australia & 218624 & $(9 \cdot 0)$ & 27 & $49 \cdot 3$ & $5 \cdot 7$ & 694 & (291-1653) & 740 & $(736-743)$ & 2085 & $(972-4417)$ \\
\hline Tasmania & 48633 & $(2 \cdot 0)$ & 24 & $47 \cdot 6$ & $1 \cdot 1$ & 483 & $(245-991)$ & 526 & $(523-531)$ & & \\
\hline Victoria & 501335 & $(20 \cdot 7)$ & 28 & $52 \cdot 3$ & $0 \cdot 5$ & 494 & (184-995) & 507 & $(506-509)$ & & \\
\hline Western Australia & 274591 & $(11 \cdot 3)$ & 25 & $50 \cdot 6$ & $18 \cdot 1$ & 684 & $(251-1262)$ & 713 & $(711-716)$ & 3353 & $(1535-4340)$ \\
\hline
\end{tabular}

CI, Confidence interval.

* Assumes all cases without Indigenous status reported were non-Indigenous.

$\dagger$ Only calculated for jurisdictions with Indigenous status reported for $>75 \%$ of notified cases (Northern Territory, South Australia, Western Australia);,$-<50$ Indigenous cases notified. 

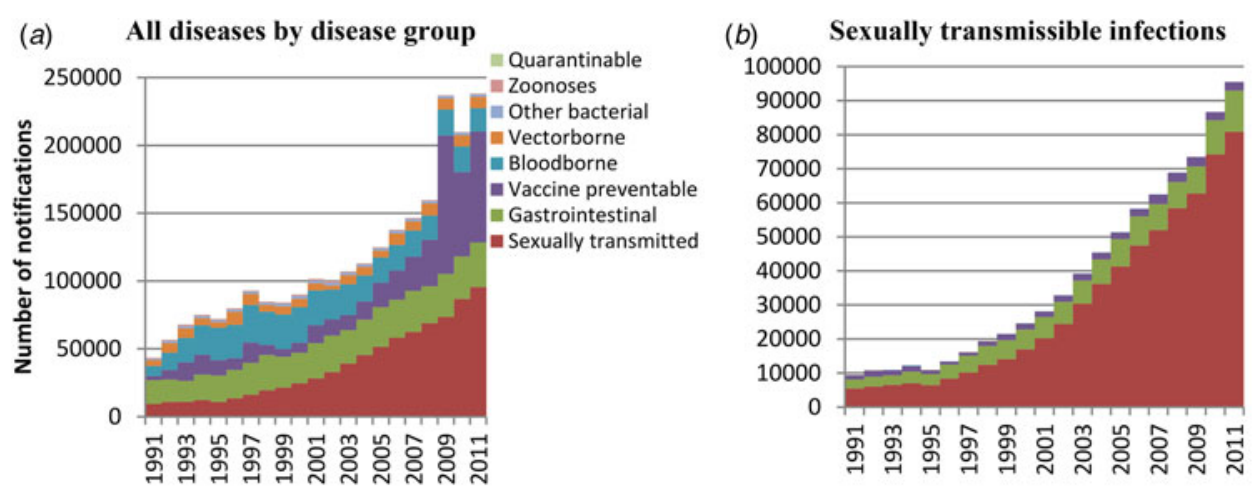

(d) Vaccine preventable diseases
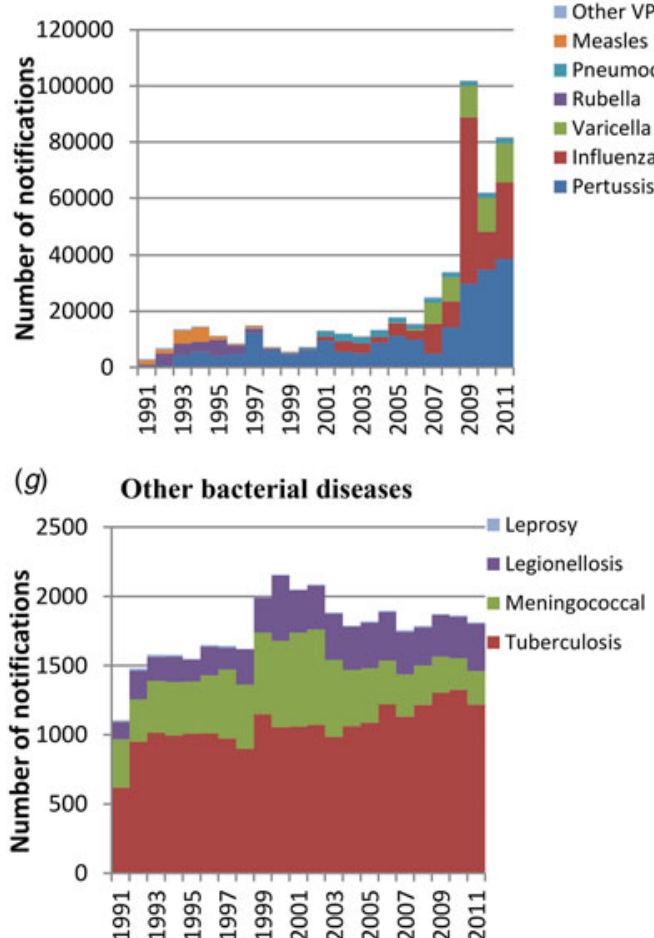

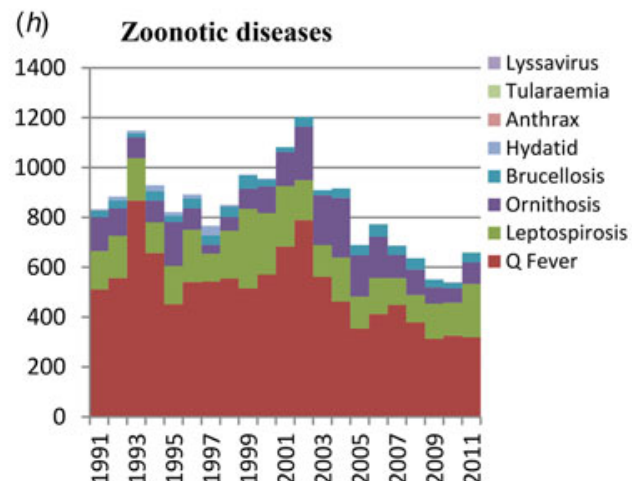

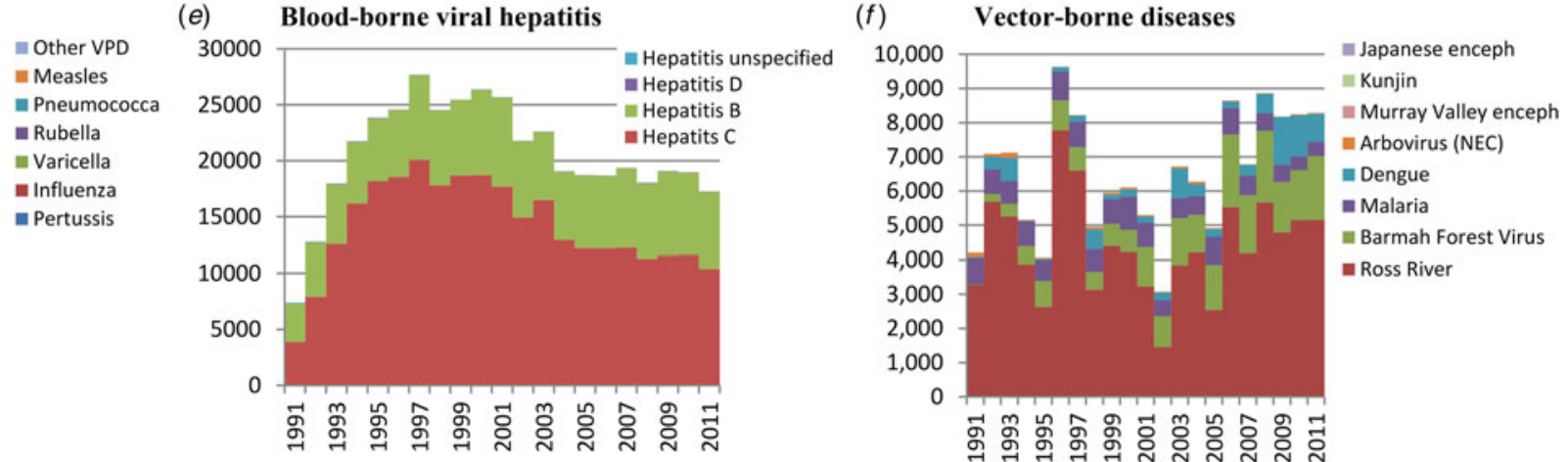

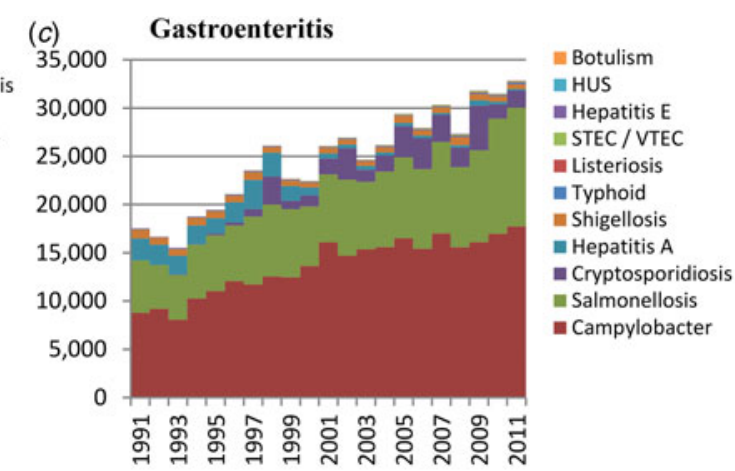

న

( $f$ ) Vector-borne diseases

(i) Quarantinable diseases

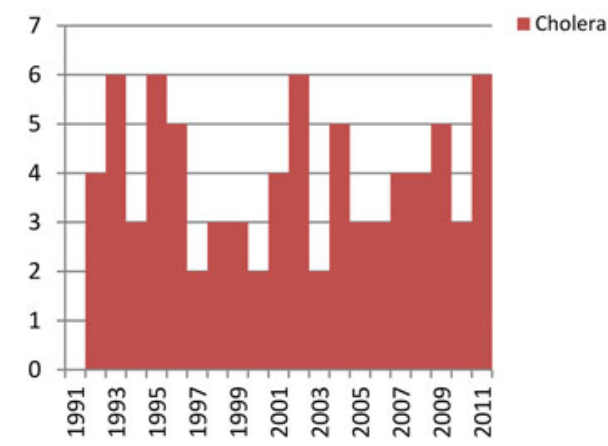

Fig. 1. Number of notifications by disease group and year, Australia 1991-2011. HUS, Haemolytic uraemic syndrome; NEC, not elsewhere classified; STEC/VTEC, Shiga/ Vero-toxin-producing Escherichia coli; VPD, vaccine-preventable disease. 
Table 3. Infectious disease notification incidence by sub-period and age group, Australia 1991-2011

\begin{tabular}{|c|c|c|c|c|c|c|c|c|c|c|c|c|c|c|c|c|c|}
\hline & \multirow{2}{*}{\multicolumn{2}{|c|}{ Overall }} & \multirow{3}{*}{$\begin{array}{l}\text { Average } \\
\text { change } \\
\text { annual } \\
\text { inc. }\end{array}$} & \multicolumn{6}{|c|}{ Sub-period } & \multicolumn{8}{|c|}{ Age group } \\
\hline & & & & \multicolumn{2}{|c|}{ 1991-1997 } & \multicolumn{2}{|c|}{ 1998-2004 } & \multicolumn{2}{|c|}{ 2005-2011 } & \multicolumn{2}{|c|}{$<5$ years } & \multicolumn{2}{|c|}{$5-19$ years } & \multicolumn{2}{|c|}{ 20-64 years } & \multicolumn{2}{|c|}{$65-98$ years } \\
\hline & notified & Inc. & & Inc. & $\mathrm{RR}$ & Inc. & $\mathrm{RR}$ & Inc. & $\mathrm{RR}$ & Inc. & $\mathrm{RR}$ & Inc. & $\mathrm{RR}$ & Inc. & $\mathrm{RR}$ & Inc. & $\mathrm{RR}$ \\
\hline All diseases & 2421134 & $591 \cdot 2$ & $6 \cdot 4 \%$ & $385 \cdot 9$ & $0 \cdot 5$ & $504 \cdot 5$ & $0 \cdot 6$ & $853 \cdot 0$ & Ref. & $698 \cdot 4$ & $1 \cdot 1$ & $564 \cdot 6$ & $0 \cdot 9$ & $653 \cdot 9$ & Ref. & $262 \cdot 6$ & $0 \cdot 4$ \\
\hline Bloodborne viral hepatitis & 431608 & $105 \cdot 6$ & $-1 \cdot 1 \%$ & $108 \cdot 6$ & $1 \cdot 2$ & $122 \cdot 7$ & $1 \cdot 4$ & $87 \cdot 4$ & Ref. & $5 \cdot 9$ & $0 \cdot 04$ & $28 \cdot 5$ & $0 \cdot 2$ & $158 \cdot 6$ & Ref. & $28 \cdot 6$ & $0 \cdot 2$ \\
\hline Hepatitis B & 134797 & $35 \cdot 8$ & $-1 \cdot 5 \%$ & $41 \cdot 2$ & $1 \cdot 3$ & $36 \cdot 0$ & $1 \cdot 1$ & $32 \cdot 5$ & & $2 \cdot 4$ & $0 \cdot 0$ & $14 \cdot 9$ & $0 \cdot 3$ & $51 \cdot 6$ & & $11 \cdot 8$ & $0 \cdot 2$ \\
\hline Hepatitis C & 296022 & $73 \cdot 9$ & $-5 \cdot 3 \%$ & $100 \cdot 2$ & $1 \cdot 8$ & $86 \cdot 9$ & $1 \cdot 6$ & $54 \cdot 8$ & & $3 \cdot 3$ & $0 \cdot 0$ & $15 \cdot 6$ & $0 \cdot 1$ & $113 \cdot 0$ & & $17 \cdot 4$ & $0 \cdot 2$ \\
\hline Hepatitis D & 573 & $0 \cdot 1$ & $3 \cdot 1 \%$ & - & - & $0 \cdot 1$ & $0 \cdot 8$ & $0 \cdot 2$ & & $0 \cdot 006$ & $0 \cdot 0$ & $0 \cdot 04$ & $0 \cdot 2$ & $0 \cdot 2$ & & $0 \cdot 02$ & $0 \cdot 1$ \\
\hline Hepatitis (NEC) & 216 & 0.06 & & & & & & & & & & & & & & & \\
\hline Gastrointestinal diseases & 518808 & $126 \cdot 7$ & $2 \cdot 0 \%$ & $105 \cdot 0$ & $0 \cdot 7$ & $129 \cdot 8$ & $0 \cdot 9$ & $142 \cdot 0$ & Ref. & $440 \cdot 4$ & $4 \cdot 2$ & $109 \cdot 4$ & $1 \cdot 1$ & $104 \cdot 2$ & Ref. & $88 \cdot 2$ & $0 \cdot 8$ \\
\hline Botulism & 16 & $0 \cdot 005$ & & & & & & & & & & & & & & & \\
\hline Campylobacteriosis & 286865 & $105 \cdot 4$ & $2 \cdot 0 \%$ & $85 \cdot 9$ & $0 \cdot 7$ & $112 \cdot 4$ & $1 \cdot 0$ & $115 \cdot 2$ & & $255 \cdot 3$ & $2 \cdot 6$ & $86 \cdot 7$ & $0 \cdot 9$ & $97 \cdot 2$ & & $89 \cdot 4$ & $0 \cdot 9$ \\
\hline Cryptosporidiosis & 33157 & 11.9 & $0 \cdot 0 \%$ & - & - & $9 \cdot 9$ & $0 \cdot 8$ & $12 \cdot 9$ & & $79 \cdot 0$ & $12 \cdot 2$ & $12 \cdot 9$ & $2 \cdot 0$ & $6 \cdot 5$ & & $1 \cdot 8$ & $0 \cdot 3$ \\
\hline Haemolytic uraemic syndrome & 260 & $0 \cdot 08$ & & & & & & & & & & & & & & & \\
\hline Hepatitis A virus & 23506 & $5 \cdot 7$ & $-12 \cdot 9 \%$ & $11 \cdot 9$ & $8 \cdot 7$ & $4 \cdot 8$ & $3 \cdot 5$ & $1 \cdot 4$ & & $4 \cdot 9$ & $0 \cdot 8$ & $7 \cdot 0$ & $1 \cdot 1$ & $6 \cdot 2$ & & $1 \cdot 5$ & $0 \cdot 2$ \\
\hline Hepatitis E virus & 346 & $0 \cdot 1$ & & & & & & & & & & & & & & & \\
\hline Listeriosis & 1295 & $0 \cdot 3$ & $0 \cdot 4 \%$ & $0 \cdot 3$ & $1 \cdot 0$ & $0 \cdot 3$ & $1 \cdot 1$ & $0 \cdot 3$ & & $0 \cdot 4$ & $1 \cdot 8$ & $0 \cdot 01$ & $0 \cdot 1$ & $0 \cdot 2$ & & $1 \cdot 3$ & $5 \cdot 9$ \\
\hline Salmonellosis & 157428 & $38 \cdot 4$ & $3 \cdot 0 \%$ & $30 \cdot 5$ & $0 \cdot 7$ & $37 \cdot 4$ & $0 \cdot 8$ & $45 \cdot 9$ & & $195 \cdot 7$ & $7 \cdot 7$ & $32 \cdot 4$ & $1 \cdot 3$ & $25 \cdot 4$ & & $24 \cdot 3$ & $1 \cdot 0$ \\
\hline Shiga/Vero-toxin-producing & 1035 & $0 \cdot 4$ & $5 \cdot 7 \%$ & - & - & $0 \cdot 3$ & $0 \cdot 6$ & $0 \cdot 5$ & & $0 \cdot 9$ & $3 \cdot 1$ & $0 \cdot 4$ & $1 \cdot 5$ & $0 \cdot 3$ & & $0 \cdot 6$ & $2 \cdot 1$ \\
\hline \multicolumn{18}{|l|}{ E. coli } \\
\hline Shigellosis & 13297 & $3 \cdot 8$ & $-5 \cdot 5 \%$ & $6 \cdot 2$ & $2 \cdot 1$ & $3 \cdot 1$ & $1 \cdot 1$ & $2 \cdot 9$ & & $17 \cdot 3$ & $5 \cdot 7$ & $3 \cdot 0$ & $1 \cdot 0$ & $3 \cdot 0$ & & $1 \cdot 2$ & $0 \cdot 4$ \\
\hline Typhoid fever & 1603 & $0 \cdot 4$ & $1 \cdot 8 \%$ & $0 \cdot 4$ & $0 \cdot 9$ & $0 \cdot 3$ & $0 \cdot 7$ & $0 \cdot 5$ & & $0 \cdot 4$ & $1 \cdot 0$ & $0 \cdot 5$ & $1 \cdot 1$ & $0 \cdot 4$ & & $0 \cdot 09$ & $0 \cdot 2$ \\
\hline Quarantinable diseases & 79 & $0 \cdot 02$ & & & & & & & & & & & & & & & \\
\hline Cholera & 79 & $0 \cdot 02$ & & & & & & & & & & & & & & & \\
\hline Sexually transmissible infections $\dagger$ & 790990 & $192 \cdot 9$ & $11 \cdot 8 \%$ & $64 \cdot 4$ & $0 \cdot 2$ & $156 \cdot 3$ & $0 \cdot 5$ & $333 \cdot 8$ & Ref. & $6 \cdot 1$ & $0 \cdot 03$ & $228 \cdot 7$ & $1 \cdot 0$ & $239 \cdot 1$ & Ref. & $11 \cdot 2$ & $0 \cdot 05$ \\
\hline Chancroid & 8 & $0 \cdot 005$ & & & & & & & & & & & & & & & \\
\hline Chlamydial infection & 621431 & $185 \cdot 1$ & $12 \cdot 5 \%$ & $64 \cdot 4$ & $0 \cdot 2$ & $119 \cdot 8$ & $0 \cdot 4$ & $280 \cdot 3$ & & $3 \cdot 3$ & $0 \cdot 01$ & $234 \cdot 3$ & $1 \cdot 0$ & $226 \cdot 7$ & & $2 \cdot 9$ & $0 \cdot 01$ \\
\hline Donovoanosis & 426 & $0 \cdot 09$ & $-17 \cdot 5 \%$ & $0 \cdot 3$ & $46 \cdot 3$ & $0 \cdot 09$ & $15 \cdot 4$ & $0 \cdot 006$ & & $0 \cdot 03$ & $0 \cdot 3$ & $0 \cdot 10$ & $0 \cdot 9$ & $0 \cdot 11$ & & $0 \cdot 02$ & $0 \cdot 2$ \\
\hline Gonococcal infection & 130008 & $31 \cdot 5$ & $5 \cdot 6 \%$ & $18 \cdot 4$ & $0 \cdot 4$ & $32 \cdot 4$ & $0 \cdot 8$ & $41 \cdot 8$ & & $1 \cdot 8$ & $0 \cdot 05$ & $33 \cdot 2$ & $0 \cdot 8$ & $40 \cdot 4$ & & $1 \cdot 4$ & $0 \cdot 03$ \\
\hline Syphilis (incl. congenital $n=54$ ) & 39117 & $9 \cdot 4$ & $3 \cdot 4 \%$ & $6 \cdot 8$ & $0 \cdot 6$ & $9 \cdot 3$ & $0 \cdot 8$ & $11 \cdot 7$ & & $0 \cdot 9$ & $0 \cdot 07$ & $5 \cdot 2$ & $0 \cdot 4$ & $12 \cdot 2$ & & $7 \cdot 1$ & $0 \cdot 6$ \\
\hline Vaccine-preventable diseases & 487176 & $119 \cdot 0$ & $14 \cdot 7 \%$ & $57 \cdot 5$ & $0 \cdot 2$ & $51 \cdot 8$ & $0 \cdot 2$ & $241 \cdot 6$ & Ref. & $229 \cdot 3$ & $2 \cdot 5$ & $178 \cdot 1$ & $2 \cdot 0$ & $90 \cdot 7$ & Ref. & $95 \cdot 9$ & $1 \cdot 1$ \\
\hline Diphtheria & 358 & $0 \cdot 09$ & & & & & & & & & & & & & & & \\
\hline Haemophilus influenzae type b & 2081 & $0 \cdot 5$ & $-24 \cdot 7 \%$ & $1 \cdot 4$ & $15 \cdot 7$ & $0 \cdot 1$ & $1 \cdot 5$ & $0 \cdot 09$ & & $5 \cdot 6$ & $75 \cdot 2$ & $0 \cdot 3$ & $3 \cdot 5$ & $0 \cdot 07$ & & $0 \cdot 2$ & $2 \cdot 6$ \\
\hline Influenza (laboratory confirmed) & 137940 & $60 \cdot 6$ & $32 \cdot 6 \%$ & - & - & $13 \cdot 4$ & $0 \cdot 2$ & $87 \cdot 8$ & & $143 \cdot 0$ & $2 \cdot 8$ & $90 \cdot 5$ & $1 \cdot 8$ & $50 \cdot 9$ & & $35 \cdot 1$ & $0 \cdot 7$ \\
\hline Measles & 16780 & $3 \cdot 9$ & $-22 \cdot 9 \%$ & $11 \cdot 6$ & $29 \cdot 8$ & $0 \cdot 7$ & $1 \cdot 8$ & $0 \cdot 4$ & & $17 \cdot 0$ & $18 \cdot 3$ & $10 \cdot 4$ & $11 \cdot 3$ & $0 \cdot 9$ & & $0 \cdot 2$ & $0 \cdot 2$ \\
\hline Mumps & 3363 & $1 \cdot 0$ & $0 \cdot 7 \%$ & $1 \cdot 0$ & $0 \cdot 8$ & $0 \cdot 7$ & 0.6 & $1 \cdot 2$ & & $1 \cdot 3$ & $1 \cdot 3$ & $1 \cdot 3$ & $1 \cdot 3$ & $1 \cdot 0$ & & $0 \cdot 2$ & $0 \cdot 2$ \\
\hline Pertussis & 220541 & $53 \cdot 9$ & $13 \cdot 4 \%$ & $25 \cdot 6$ & $0 \cdot 3$ & $33 \cdot 4$ & $0 \cdot 3$ & $96 \cdot 3$ & & $86 \cdot 6$ & $2 \cdot 1$ & $87 \cdot 3$ & $2 \cdot 1$ & $41 \cdot 8$ & & $39 \cdot 3$ & $0 \cdot 9$ \\
\hline Pneumococcal disease (invasive) & 21813 & $8 \cdot 8$ & $-4 \cdot 5 \%$ & & & $11 \cdot 2$ & $1 \cdot 5$ & $7 \cdot 6$ & & $31 \cdot 5$ & $5 \cdot 3$ & $3 \cdot 0$ & $0 \cdot 5$ & $6 \cdot 0$ & & $19 \cdot 9$ & $3 \cdot 3$ \\
\hline
\end{tabular}




\begin{tabular}{|c|c|c|c|c|c|c|c|c|c|c|c|c|c|c|c|c|c|}
\hline & \multirow{2}{*}{\multicolumn{2}{|c|}{ Overall }} & \multirow{3}{*}{$\begin{array}{l}\text { Average } \\
\text { change } \\
\text { annual } \\
\text { inc. }\end{array}$} & \multicolumn{6}{|c|}{ Sub-period } & \multicolumn{8}{|c|}{ Age group } \\
\hline & & & & \multicolumn{2}{|c|}{ 1991-1997 } & \multicolumn{2}{|c|}{ 1998-2004 } & \multicolumn{2}{|c|}{ 2005-2011 } & \multicolumn{2}{|c|}{$<5$ years } & \multicolumn{2}{|c|}{$5-19$ years } & \multicolumn{2}{|c|}{ 20-64 years } & \multicolumn{2}{|c|}{$65-98$ years } \\
\hline & $\begin{array}{l}\text { Cases } \\
\text { notified }\end{array}$ & Inc. & & Inc. & $\mathrm{RR}$ & Inc. & $\mathrm{RR}$ & Inc. & $\mathrm{RR}$ & Inc. & $\mathrm{RR}$ & Inc. & $\mathrm{RR}$ & Inc. & $\mathrm{RR}$ & Inc. & $\mathrm{RR}$ \\
\hline Poliomyelitis & 1 & $0 \cdot 000$ & & & & & & & & & & & & & & & \\
\hline Rubella (incl. congenital $n=4$ ) & 24388 & $5 \cdot 3$ & $-30 \cdot 4 \%$ & $19 \cdot 5$ & $99 \cdot 5$ & $1 \cdot 5$ & $7 \cdot 7$ & $0 \cdot 2$ & & $6 \cdot 5$ & $1 \cdot 6$ & $11 \cdot 6$ & $2 \cdot 9$ & $4 \cdot 0$ & & $0 \cdot 3$ & $0 \cdot 07$ \\
\hline Tetanus & 120 & $0 \cdot 03$ & & & & & & & & & & & & & & & \\
\hline Varicella zoster & 59791 & $77 \cdot 9$ & $4 \cdot 1 \%$ & - & - & - & - & $77 \cdot 9$ & & $91 \cdot 6$ & $1 \cdot 5$ & $92 \cdot 0$ & $1 \cdot 5$ & $62 \cdot 1$ & & $123 \cdot 2$ & $2 \cdot 0$ \\
\hline Vector-borne diseases & 137817 & $33 \cdot 7$ & $0 \cdot 5 \%$ & $36 \cdot 1$ & $1 \cdot 0$ & $28 \cdot 5$ & $0 \cdot 8$ & $36 \cdot 3$ & Ref. & $3 \cdot 2$ & $0 \cdot 07$ & $13 \cdot 3$ & $0 \cdot 3$ & $46 \cdot 6$ & Ref. & $21 \cdot 1$ & $0 \cdot 5$ \\
\hline Arbovirus infection (NEC) & 869 & $0 \cdot 2$ & $-9 \cdot 1 \%$ & $0 \cdot 3$ & $3 \cdot 7$ & $0 \cdot 3$ & $2 \cdot 9$ & $0 \cdot 09$ & & $0 \cdot 02$ & $0 \cdot 06$ & $0 \cdot 08$ & $0 \cdot 3$ & $0 \cdot 3$ & & $0 \cdot 2$ & $0 \cdot 6$ \\
\hline Barmah Forest virus infection & 21815 & $6 \cdot 1$ & $6 \cdot 0 \%$ & $4 \cdot 3$ & $0 \cdot 5$ & $4 \cdot 7$ & $0 \cdot 6$ & $8 \cdot 1$ & & $0 \cdot 2$ & $0 \cdot 03$ & $1 \cdot 8$ & $0 \cdot 2$ & $8 \cdot 4$ & & $5 \cdot 1$ & $0 \cdot 6$ \\
\hline Dengue virus infection & 8691 & $2 \cdot 1$ & $8 \cdot 1 \%$ & $1 \cdot 2$ & $0 \cdot 4$ & $1 \cdot 9$ & $0 \cdot 6$ & $3 \cdot 2$ & & $0 \cdot 3$ & $0 \cdot 09$ & $1 \cdot 1$ & $0 \cdot 4$ & $2 \cdot 9$ & & $1 \cdot 1$ & $0 \cdot 4$ \\
\hline Japanese encephalitis & 11 & $0 \cdot 001$ & & & & & & & & & & & & & & & \\
\hline Kunjin virus infection & 60 & $0 \cdot 02$ & & & & & & & & & & & & & & & \\
\hline Malaria & 13733 & $3 \cdot 3$ & $-3 \cdot 3 \%$ & $4 \cdot 0$ & $1 \cdot 5$ & $3 \cdot 4$ & $1 \cdot 3$ & $2 \cdot 7$ & & $1 \cdot 8$ & $0 \cdot 5$ & $3 \cdot 5$ & $0 \cdot 9$ & $4 \cdot 0$ & & $0 \cdot 8$ & $0 \cdot 2$ \\
\hline Murray Valley encephalitis virus & 79 & $0 \cdot 01$ & & & & & & & & & & & & & & & \\
\hline Ross River virus infection & 92559 & $22 \cdot 3$ & $-1 \cdot 0 \%$ & $28 \cdot 7$ & $1 \cdot 3$ & $18 \cdot 1$ & $0 \cdot 8$ & $22 \cdot 2$ & & $0 \cdot 7$ & $0 \cdot 02$ & $7 \cdot 0$ & $0 \cdot 2$ & $31 \cdot 5$ & & $14 \cdot 5$ & $0 \cdot 5$ \\
\hline Zoonoses & 17696 & $4 \cdot 4$ & $-3 \cdot 2 \%$ & $5 \cdot 1$ & $1 \cdot 6$ & $5 \cdot 1$ & $1 \cdot 7$ & $3 \cdot 1$ & Ref. & $0 \cdot 3$ & $0 \cdot 04$ & $1 \cdot 8$ & $0 \cdot 3$ & $6 \cdot 0$ & Ref. & $2 \cdot 7$ & $0 \cdot 4$ \\
\hline Anthrax & 4 & $0 \cdot 001$ & & & & & & & & & & & & & & & \\
\hline Australian bat lyssavirus & 1 & $0 \cdot 0$ & & & & & & & & & & & & & & & \\
\hline Brucellosis & 724 & $0 \cdot 2$ & $0 \cdot 3 \%$ & $0 \cdot 2$ & $1 \cdot 0$ & $0 \cdot 2$ & $1 \cdot 0$ & $0 \cdot 2$ & & $0 \cdot 01$ & $0 \cdot 04$ & $0 \cdot 08$ & $0 \cdot 3$ & $0 \cdot 3$ & & $0 \cdot 07$ & $0 \cdot 3$ \\
\hline Hydatid infection & 136 & $0 \cdot 07$ & & & & & & & & & & & & & & & \\
\hline Leptospirosis & 3544 & $0 \cdot 9$ & $-2 \cdot 1 \%$ & $0 \cdot 9$ & $1 \cdot 3$ & $1 \cdot 1$ & $1 \cdot 7$ & $0 \cdot 7$ & & $0 \cdot 02$ & $0 \cdot 02$ & $0 \cdot 4$ & $0 \cdot 3$ & $1 \cdot 2$ & & $0 \cdot 3$ & $0 \cdot 3$ \\
\hline Ornithosis & 2480 & $0 \cdot 8$ & $-4 \cdot 5 \%$ & $0 \cdot 9$ & 1.9 & $1 \cdot 0$ & $2 \cdot 0$ & $0 \cdot 5$ & & $0 \cdot 06$ & $0 \cdot 06$ & $0 \cdot 09$ & $0 \cdot 10$ & $1 \cdot 0$ & & $1 \cdot 3$ & $1 \cdot 3$ \\
\hline Q fever & 10805 & $2 \cdot 6$ & $-4 \cdot 1 \%$ & $3 \cdot 3$ & 1.9 & $3 \cdot 1$ & $1 \cdot 8$ & $1 \cdot 7$ & & $0 \cdot 2$ & $0 \cdot 04$ & $1 \cdot 2$ & $0 \cdot 3$ & $3 \cdot 7$ & & $1 \cdot 2$ & $0 \cdot 3$ \\
\hline Tularaemia & 2 & $0 \cdot 001$ & & & & & & & & & & & & & & & \\
\hline Other bacterial diseases & 36960 & $9 \cdot 0$ & $0 \cdot 1 \%$ & $8 \cdot 7$ & $1 \cdot 0$ & $10 \cdot 1$ & $1 \cdot 2$ & $8 \cdot 8$ & Ref. & $13 \cdot 2$ & $1 \cdot 5$ & $4 \cdot 9$ & $0 \cdot 6$ & $8 \cdot 8$ & Ref. & $14 \cdot 8$ & $1 \cdot 7$ \\
\hline Legionellosis & 5658 & $1 \cdot 4$ & $2 \cdot 3 \%$ & $1 \cdot 0$ & $0 \cdot 6$ & $1 \cdot 7$ & $1 \cdot 1$ & $1 \cdot 5$ & & $0 \cdot 03$ & $0 \cdot 02$ & $0 \cdot 06$ & $0 \cdot 04$ & $1 \cdot 3$ & & $4 \cdot 7$ & $3 \cdot 6$ \\
\hline Leprosy & 200 & $0 \cdot 05$ & & & & & & & & & & & & & & & \\
\hline Meningococcal disease (invasive) & 8766 & $2 \cdot 1$ & $-2 \cdot 7 \%$ & $2 \cdot 2$ & $1 \cdot 6$ & $3 \cdot 0$ & $2 \cdot 2$ & $1 \cdot 4$ & & $11 \cdot 5$ & $11 \cdot 0$ & $3 \cdot 1$ & $3 \cdot 0$ & $1 \cdot 0$ & & $0 \cdot 8$ & $0 \cdot 8$ \\
\hline Tuberculosis & 22336 & $5 \cdot 5$ & $0 \cdot 4 \%$ & $5 \cdot 4$ & $1 \cdot 0$ & $5 \cdot 4$ & $0 \cdot 9$ & $5 \cdot 7$ & & $1 \cdot 7$ & $0 \cdot 3$ & $1 \cdot 7$ & $0 \cdot 3$ & $6 \cdot 4$ & & $9 \cdot 3$ & $1 \cdot 4$ \\
\hline
\end{tabular}

Inc., Incidence per 100000 person-years; Avg change inc., \% average percentage change in annual incidence per year of the study period (while disease was nationally notifiable; Table 1); RR, relative risk; Ref., reference group for RR calculations; NEC, not elsewhere classified; -, not notifiable for that sub-period.

Annual change in notification incidence, sub-period and age-group analysis for diseases with $>400$ cases notified.

$\dagger$ Chlamydial and gonococcal infections and syphilis include non-sexually acquired infections (especially in children) such as perinatal and eye infections.

Diseases with zero notifications: highly pathogenic avian influenza in humans (HPAIH), plague, rabies, severe acute respiratory syndrome (SARS), smallpox, viral haemorrhagic fevers, yellow fever. 


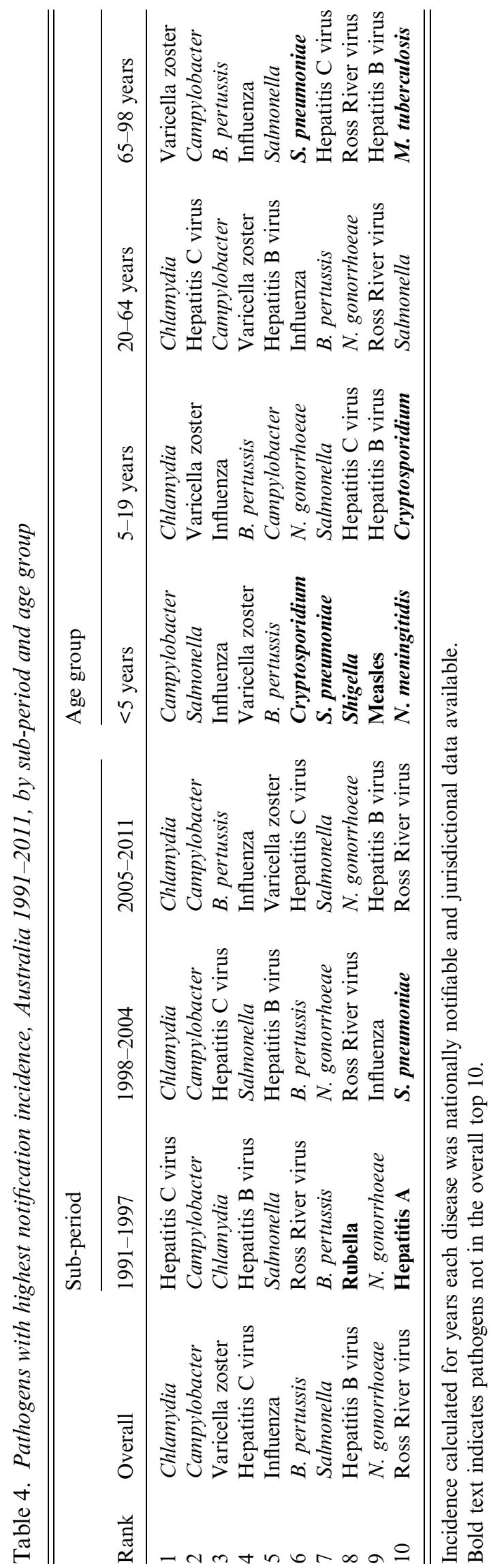

increase/year, notifiable from 2001), pertussis (13\%), and chlamydial infection (13\%, notifiable from 1994) increased the most across the study period. Twentyone diseases were consistently notifiable across jurisdictions for the entire study period (Table 1); annual incidence of these increased by $4 \cdot 1 \%$ per year.

The median age of notified cases was 27 [interquartile range (IQR) 19-40] years, younger for STIs and in the NT (both with median age 23 years) (Table 2). Median age at onset was $\leqslant 7$ years for congenital rubella, congenital syphilis, botulism, Hib, cryptosporidiosis, chickenpox and haemolytic uraemic syndrome; and $\geqslant 62$ years for legionellosis, listeriosis and tetanus. Notification incidence (/100000 per year) was highest for young children aged $<5$ years (698) and adults aged 20-64 years (654) and lowest for older adults aged 65-98 years (263) (Table 3). Cryptosporidiosis, invasive pneumococcal disease, shigellosis, measles and invasive meningococcal disease were among the 10 highest-incidence diseases for young children; cryptosporidiosis for older children and adolescents (aged 5-19 years); and pneumococcal disease and tuberculosis for older adults (Table 4). Compared to adults (aged 20-64 years), notification RR was highest for Hib (75), measles (18), cryptosporidiosis (12) and invasive meningococcal disease (11) for young children; measles (11) for older children and adolescents; and listeriosis (6) for older adults (Table 3). Fifty-one percent of notified cases were male, ranging from $46 \%$ for STIs to $79 \%$ for zoonoses (Table 2).

Overall, $202584(8.4 \%)$ cases were identified as Indigenous - ranging from $0.5 \%$ in the Australian Capital Territory (ACT) and Victoria to $58 \%$ in the NT (Table 2) $-36 \%$ of cases identified as nonIndigenous and for 56\% Indigenous status was not reported. STIs comprised $70 \%$ of Indigenous case notifications. In the three jurisdictions with Indigenous status completed for $>75 \%$ of cases, notification rates for all diseases were six times higher and STIs 14 times higher in Indigenous Australians compared to the total population.

Queensland had the greatest number of notifications (644 556 notifications, 27\%), despite ranking third in population behind NSW and Victoria. The NT had the highest annual notification incidence (2598/100 000) (Fig. 2); age-standardized notification rates remained four times higher in the NT than the national average (2512 vs. 621/100000 per year). Notification rates were highest in the NT for all disease groups except quarantinable and zoonotic diseases; however, the NT made the best progress in 

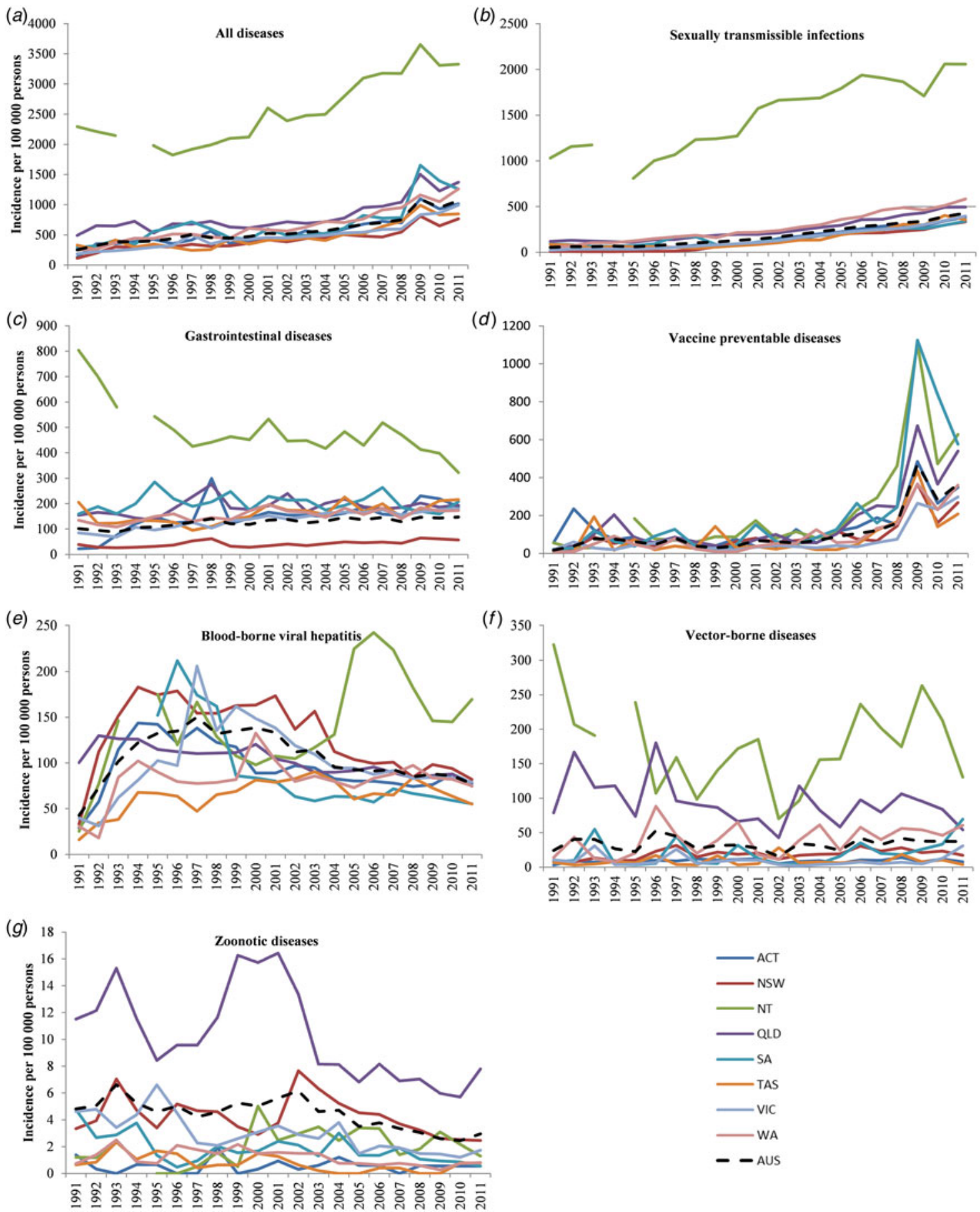

Fig. 2. Annual notification rate by jurisdiction and year for all diseases groups, Australia 1991-2011. NT notifications from 1994 not included in incidence calculations.

reducing (or limiting increase of) notifications overall (Supplementary Appendix Fig. A1). Compared to notification incidence in NSW, RR was $\geqslant 15$ for shigellosis, gonococcal infection, and syphilis in the NT; brucellosis in Queensland; and Shiga- or Verotoxin-producing Escherichia coli (STEC/VTEC) in SA (Supplementary Appendix Table A1).

\section{STIs}

STIs comprised $33 \%$ of all notifications, increasing fivefold from 64/100 000 per year in 1991-1997 to $334 / 100000$ per year in 2005-2011 (Table 3). Chlamydial infections (notifiable from 1994) accounted for $79 \%$ of STI notifications, gonococcal 
disease $16 \%(32 / 100000$ per year), and syphilis $5 \%(9 / 100000$ per year) (Fig. 1b). Annual incidence of STIs was highest in those aged 20-29 years $(239 / 100000)$ and was eightfold higher in the NT than the national average (1527 vs. 193/100 000) (Fig. 2b).

\section{Gastrointestinal diseases}

Gastrointestinal diseases comprised $21 \%$ of notifications, with campylobacteriosis accounting for 55\% despite not being notifiable in NSW (Fig. 1c). The national incidence of notified gastrointestinal diseases increased by $2 \%$ per year. Gastrointestinal disease notifications in the NT were nearly four times the national incidence (481 vs. 127/100000 per year, Fig. 2c). NSW had the lowest notification incidence (Supplementary Appendix Fig. A1); however, national notification incidence of gastrointestinal diseases excluding campylobacteriosis (55/100 000 per year) was similar to NSW (43/100 000 per year).

\section{VPDs}

Just under half a million VPD cases were notified (Table 2), of which $45 \%$ were pertussis, $28 \%$ influenza (notifiable from 2001), and 12\% varicella zoster (notifiable from 2006) (Fig. 1d). VPD notifications increased $15 \%$ per year, from 3016 in 1991 to a peak of 101942 cases in 2009 during the H1N1 influenza pandemic. Median age at disease onset increased from 14 years in 1991-1997 to 30 years in 2005-2011. National incidence of VPD notifications was $119 / 100000$ per year, highest in the NT $(239 / 100000)$ and lowest in Victoria (77/100 000) (Fig. 2d).

\section{BBVH}

BBVH comprised $18 \%$ of notifications nationally and $30 \%$ of notifications in NSW (Supplementary Appendix Fig. A1). Annual incidence of notified BBVH cases was higher in the NT and NSW (144 and 128 notifications $/ 100000$, respectively) than other jurisdictions (64-101/100 000, Fig. 2e). Hepatitis C, notifiable from 1995, accounted for $69 \%$ and hepatitis B 31\% (Fig. 1e). BBVH notification incidence increased threefold from 43/100 000 per year persons in 1991 to $150 / 100000$ per year in 1997 before dropping to $77 / 100000$ per year in 2011, reflecting changes in hepatitis $\mathrm{C}$ notifications.

\section{VBDs}

There were 137817 VBD notifications (5.7\% of notifications), $67 \%$ being RRV infections (notifiable from 1993), 16\% Barmah Forest virus infections (notifiable from 1995), 10\% malaria, and 6\% dengue (Fig. 1f). VBD notification incidence was 34/100000 per year for Australia, highest in the NT (175/100 000) and Queensland (92/100000) and lowest in Tasmania (9/100 000) (Fig. 2f).

\section{Other bacterial diseases}

There were 36960 notifications of other bacterial diseases, of which $60 \%$ were tuberculosis, $24 \%$ invasive meningococcal disease, and 15\% legionellosis. Other bacterial disease notification incidence was $9 / 100000$ per year, highest in the NT (27/100 000) and lowest in Tasmania (5/100000) (Fig. 2). Tuberculosis notification incidence was $6 / 100000$ per year for Australia (range 21/100000 in the NT to $2 / 100000$ in Tasmania) and was stable over the study period (Supplementary Appendix Fig. A1). Fifty-five percent of notified cases were male; highest for Legionella $(67 \%)$ and leprosy $(62 \%)$.

\section{Zoonotic diseases}

There were 17696 zoonotic disease notifications $(0 \cdot 7 \%$ of all notifications). Q fever was most common (61\%), followed by leptospirosis $(20 \%)$ and ornithosis (14\%). Males predominated, especially for anthrax $(100 \%$ of four cases notified), leptospirosis $(90 \%)$, brucellosis $(84 \%)$, and Q fever (80\%). Queensland notified $43 \%$ of all zoonotic cases, $83 \%$ of brucellosis and $56 \%$ of leptospirosis cases. Zoonotic disease notification incidence was $4 \cdot 4 / 100000$ per year; highest in Queensland (10.0/100000) and lowest in the ACT $(0 \cdot 6 / 100000)$ (Fig. 2h). Zoonotic disease notifications fell by an average 3\% annually (Supplementary Appendix Fig. A1).

\section{DISCUSSION}

Intelligence obtained from national communicable disease surveillance regarding infectious disease epidemiology guides national policy development, resource allocation, disease control programmes and quarantine activities, as well as allowing identification of and coordinated responses to national or multijurisdictional outbreaks [3]. This paper provides the first trend analysis of all nationally notifiable diseases in 
Australia (except HIV/AIDS and CJD) since the inception of the NNDSS in 1991. Both the number and incidence of notifications increased steadily over the 21 years, partly due to the addition of diseases to the system. Incidence rates were highest in the NT and in Indigenous and young Australians. The ten diseases with highest notification incidence accounted for nearly $90 \%$ of notifications and required a range of public health strategies for disease prevention and control; including safe sex, contact tracing, harmreduction for people who inject drugs, food safety, and immunization [10-14]. This highlights the complex challenges facing state, territory, and federal health departments in preventing and controlling infectious diseases in Australia.

Indigenous people comprised $8 \%$ of notified cases but only $3 \%$ of the Australian population [15]. Significant under-reporting of Indigenous status among notified cases means likely underestimation of this proportion. Indigenous Australians have poorer health outcomes: life expectancy is 9.7 and 11.5 years lower for Indigenous females and males, respectively [16], and disease burden measured in years of life lost was 2.6 times that of non-Indigenous Australians for all causes and 3.8 times for infections in 2010 [17]. Childhood vaccination coverage is lower for Indigenous children [18], potentially explaining some difference in VPD notification rates. In a previously published study, the notification RR for Indigenous compared to non-Indigenous Australians from 2000 to 2009 was 24 for chlamydial infection and 174 for gonococcal infection [19], and higher positivity rates for Indigenous patients tested for Chlamydia confirm greater STI burden rather than ascertainment bias [20].

Higher notification rates in the NT reinforce previous findings that the health adjusted life expectancy for the NT population was 5 years less than the Australian average in 2003 (67.7 vs. 72.9 years) [21]. The NT is the least populous Australian jurisdiction (estimated resident population 231000 in 2011) [5]. The NT population is younger and has a high proportion of Indigenous persons (30\% in NT vs. 3\% nationally) than other jurisdictions [15, 22]; factors reflected in NT notifications. However, age-standardized notification rates were higher in the NT than elsewhere and notification rates in Indigenous people were higher in the NT than SA and WA. As NT data from 1994 were excluded from both the numerator (number of cases notified) and denominator (population) of incidence calculations, this would not have substantially impacted our RR calculations comparing notification incidence between the NT and other jurisdictions. It is likely that climactic and environmental diseases also impact disease incidence in the NT, while variability in health-seeking, diagnostic and notification practices might further contribute to the interjurisdictional differences.

Importantly, our results highlight some major public health achievements. The marked reduction in notification incidence for rubella, measles and $\mathrm{Hib}$ demonstrate the impact of Australia's National Immunization Programme. In 2014 Australia was recognized by the WHO to have eliminated measles [23]. Similarly, the reduction in donovanosis cases results from sustained public health programmes such as the National Donovanosis Eradication Project [24].

The annual number of notifications increased more than fivefold over the 21-year study period. The reasons for this are multifactorial, including addition of notifiable diseases to national and jurisdictional notification lists, population growth, introduction of screening programmes (e.g. for chlamydial infection), and improved diagnostics as well as true changes in disease incidence. There were 37 nationally notifiable diseases in 1991 and 65 in 2011. The number of nationally notifiable diseases has also increased internationally; from 56 to 87 (1992-2011) in the United States and from 41 to 58 (1991-2011) in Canada [25, 26]. The number of nationally notifiable diseases varied between European countries, ranging from 26 in France to 82 in Hungary in 2005 [27]. Some differences are due to increased number of disease categories associated with a single pathogen (e.g. syphilis has eight categories in the US system and three in the Australian NNDSS), but also reflect inclusion of diseases that are endemic in selected countries (particularly VBDs).

While many diseases are common between national surveillance systems, some differences are seen. In Australia, 10 diseases accounted for $88 \%$ of NNDSS notifications. National surveillance systems in the United States and Canada as well as the European Surveillance System (TESSy) receive notifications for eight of these diseases; varicella is not notifiable in to the Canadian or European surveillance systems and RRV is not notifiable to any [25-28]. In New Zealand, chlamydial infection, influenza, varicella and RRV are not nationally notifiable [29]. Conversely, some common infectious diseases are not notifiable in Australia and their inclusion on the Australian 
NNDL may increase the burden on notifiers and public health departments. For example, rotavirus and norovirus diseases have high notification rates in Germany [30, 31]. National notification rates for all diseases or by disease group are generally not published and the variable inclusion of high-incidence diseases limit direct comparisons, but different notification rates for individual diseases could indicate differential disease burden between countries.

A major limitation of notification data is that they underestimate the number of infections, particularly for diseases that cause mild or no clinical symptoms. Despite uniform national case definitions [32], disease notification rates are influenced by jurisdictional and local diagnostic, screening, case follow-up, and notification practices. For example, disproportionately high STEC notification rates in SA have been linked to differences in diagnostic practices with a very high number of STEC toxin gene tests performed in SA [33]. Additionally, over the 21-year study period, sensitive diagnostic tests (particularly PCR) have become widely available and marked changes to testing practices among doctors and laboratories have been documented [34-37]. These factors potentially account for much of the observed change for several diseases, including chlamydial infection, influenza and pertussis which had the greatest increase in notification incidence over the study period [34, 36, 37]. As notification fractions vary between diseases, jurisdictions, population subgroups and over time, notification rates represent the frequency of disease diagnosis but not necessarily disease incidence. Similarly, as it is impossible to determine disease severity, notification incidence alone cannot determine the population burden of infectious diseases. A European study of seven infectious diseases found foodborne diseases (campylobacteriosis and salmonellosis) had the highest notified incidence, tuberculosis the highest mortality, and HIV infection and tuberculosis the greatest disability-adjusted life years burden [38].

The NNDSS dataset provides comprehensive coverage of national infectious disease notifications over two decades. Previously, annual Australian and international reports have been produced but this paper is unique in reporting the entire dataset of nationally notifiable diseases (excluding HIV/AIDS and CJD) for a 21-year period across all Australian jurisdictions. While we provide an overview of diseases reported, we have not reported system performance or data completeness and quality. However, this analysis highlights the breadth of diseases notified in
Australia and complexity of public health responses required to reduce associated morbidity and mortality. Understanding the increasing number of notifiable diseases and notified cases is crucial for informing surveillance and public health workforce planning at a jurisdictional and national level.

\section{ACKNOWLEDGEMENTS}

The Communicable Diseases Network Australia provided the data to the project team as well as a steering committee that oversaw the project's progress and approved publication of this manuscript. The data on which this study is based is the work of many people across Australia. The authors acknowledge the contributions of public health laboratories, state and territory communicable disease control units, public health units and the Office of Health Protection.

K.G., A.C. and K.L. received NHMRC funding. K.G. received a Faculty of Medicine, Nursing and Health Sciences, Monash University scholarship.

\section{SUPPLEMENTARY MATERIAL}

For supplementary material accompanying this paper visit http://dx.doi.org/10.1017/S0950268816001072.

\section{DECLARATION OF INTEREST}

All authors: no conflict of interest that influenced writing of this paper. K. Gibney received the NHMRC Gustav Nossal Postgraduate Scholarship sponsored by CSL in 2012. This award is peer reviewed through the standard NHMRC peer review process; CSL does not play any part in the selection of the awardee. K. Leder has received travel support to attend international travel medicine conferences from Sanofi and GSK, which is unrelated to this manuscript.

\section{REFERENCES}

1. Lee L, Thacker S. The cornerstone of public health practice: public health surveillance, 1961-2011. Morbidity and Mortality Weekly Report. Surveillance Summaries 2011; 60 (Suppl. 4): 15-21.

2. Hall R. Notifiable diseases surveillance, 1917 to 1991 Communicable Diseases Intelligence 1993; 17: 226-236.

3. NNDSS Annual Report Writing Group. Australia's notifiable disease status, 2012: Annual report of the National Notifiable Diseases Surveillance System. Communicable Diseases Intelligence 2015; 39: E46-136. 
4. Australian Government. National Health Security (National Notifiable Diseases List) Instrument 2008.

5. Australian Bureau of Statistics. 3101.0-Australian Demographic Statistics: Table 59. Estimated resident population by single year of age, Australia, September 2012. Released 28 March 2013.

6. Australian Government Department of Health. National Notifiable Diseases Surveillance System-Notifications for all diseases by State \& Territory and year. 2015 (www9.health.gov.au/cda/source/rpt_2_sel_a.cfm). Accessed August 2015.

7. Ahmad O, et al. Age standardization of rates: a new WHO standard. GPE Discussion Paper Series: No. 31. 2001.

8. Australian Bureau of Statistics. 3238.0. Estimates and projections, Aboriginal and Torres Strait Islander Australians, 2001 to 2026 (www.abs.gov.au/AUS STATS/abs@.nsf/DetailsPage/3238.02001\%20to\%202026? OpenDocument). Accessed September 2014.

9. Australian Bureau of Statistics. 3238.0. Experimental estimates and projections, Aboriginal and Torres Strait Islander Australians, 1991 to 2021 (www.abs. gov.au/AUSSTATS/abs@.nsf/DetailsPage/3238.01991\%20 to\%202021? OpenDocument). Accessed November 2015.

10. Australian Government, Department of Health. Third national sexually transmissible infections strategy. Canberra: Commonwealth of Australia, 2014.

11. Australian Government, Department of Health. Fourth national hepatitis $\mathrm{C}$ strategy. Canberra: Commonwealth of Australia, 2014.

12. Australian Government, Department of Health. Second national hepatitis B strategy. Canberra: Commonwealth of Australia, 2014.

13. Australian Government, Department of Health. The Australian Immunisation Handbook, 10th edn, 2013. Canberra: Australian Government, 2013 (updated January 2014 and March 2015).

14. Communicable Diseases Network Australia. Series of National Guidelines (SoNGs); 2016 (www.health.gov. au/cdnasongs). Accessed April 2016.

15. Australian Institute of Health and Welfare. Indigenous health. Australia's health 2014; 2014 (www.aihw.gov. au/australias-health/2014/indigenous-health/). Accessed November 2015.

16. Australian Institute of Health and Welfare. The health and welfare of Australia's Aboriginal and Torres Strait Islander people. An overview, 2011. Canberra, 2011.

17. Australian Institute of Health and Welfare. Australian Burden of Disease Study: Fatal burden of disease in Aboriginal and Torres Strait Islander people, 2010. Canberra: AIHW, 2015.

18. Hull B, et al. Immunisation coverage, 2012. Communicable Diseases Intelligence Quarterly Report 2014; 38: e208-223.

19. Graham S, et al. Epidemiology of chlamydia and gonorrhoea among Indigenous and non-Indigenous Australians, 2000-2009. Medical Journal of Australia 2012; 197: 642-646.

20. Ward J, et al. Chlamydia among Australian Aboriginal and/or Torres Strait Islander people attending sexual health services, general practices and Aboriginal community controlled health services. BMC Health Services Research 2014; 285.

21. Begg S, et al. The burden of disease and injury in Australia 2003. Canberra: Australian Institute of Health and Welfare, 2007.

22. Australian Bureau of Statistics. 1362 7 . Regional statistics, Northern Territory, March 2011. Released at 11:30 am (Canberra time) 24 March 2011 (www.abs. gov.au/ausstats/abs@.nsf/Latestproducts/1362.7Feature $\% 20$ Article1Mar\%202011?opendocument\#Age). Accessed November 2014.

23. World Health Organisation Western Pacific Region. Four Western Pacific countries and areas are the first in their Region to be measles-free, 2014 (www.wpro. who.int/mediacentre/releases/2014/20140320/en/). Accessed November 2015.

24. Davis A, et al. National donovanosis eradication (elimination) project 2001-2004. Northern Territory Disease Control Bulletin 2004; 11: 26-29.

25. Centers for Disease Control and Prevention. Current and historical public health surveillance condition list with shading to indicate nationally notifiable conditions by year, 1992-2014. 2014 (wwwn.cdc.gov/nndss/script/ downloads.aspx). Accessed November 2014.

26. Public Health Agency of Canada. List of nationally notifiable diseases. 2014 (dsol-smed.phac-aspc.gc.ca/ dsol-smed/ndis/list-eng.php). Accessed November 2014.

27. Reintjesm R, et al. Benchmarking national surveillance systems: a new tool for the comparison of communicable disease surveillance and control in Europe. European Journal of Public Health 2007; 17: 375-380.

28. European Centre for Disease Prevention and Control. Annual epidemiological report, 2013. Reporting on 2011 surveillance data and 2012 epidemic intelligence data. Stockholm: ECDC, 2013.

29. New Zealand Ministry of Health. Scedule of notifiable diseases - updated October 2013, 2013 (www.health. govt.nz/our-work/diseases-and-conditions/notifiablediseases). Accessed November 2014.

30. Bernard H, et al. Epidemiology of norovirus gastroenteritis in Germany 2001-2009: eight seasons of routine surveillance. Epidemiology and Infection 2014; 142: 63-74.

31. Koch J, Wiese-Posselt M. Epidemiology of rotavirus infections in children less than 5 years of age: Germany, 2001-2008. Pediatric Infectious Diseases Journal 2011; 30: 112-117.

32. Australian Government Department of Health, Communicable Diseases Network Australia. Australian national notifiable diseases and case definitions, 2014 (www.health.gov.au/internet/main/publishing.nsf/Content/ cdna-casedefinitions.html). Accessed December 2014.

33. Combs B, Raupach JK, Kirk MD. Surveillance of Shiga toxigenic Escherichia coli in Australia. Communicable Diseases Intelligence Quarterly Report 2005; 29: 366-369.

34. Cretikos M, et al. Testing-adjusted chlamydia notification trends in New South Wales, Australia, 2000 to 
2010. Western Pacific Surveillance and Response Journal 2014; 5: 7-17.

35. Donovan B, et al. Increased testing for Neisseria gonorrhoeae with duplex nucleic acid amplification tests in Australia: implications for surveillance. Sexual Health 2015; 12: 48-50.

36. Kaczmarek M, et al. Sevenfold rise in likelihood of pertussis test requests in a stable set of Australian general practice encounters, 2000-2011. Medical Journal of Australia 2013; 198: 624-628.

37. Kelly H, et al. The significance of increased influenza notifications during spring and summer of 2010-11 in Australia. Influenza and Other Respiratory Viruses 2013; 7: 1136-1141.

38. van Lier E, Havelaar A, Nanda A. The burden of infectious diseases in Europe: a pilot study. Eurosurveillance 2007; 12: pii $=751$. 